\title{
Threshold-Based Selective Feedback for Opportunistic Interference Alignment
}

\author{
Zhinan Xu, Mingming Gan, Thomas Zemen \\ FTW (Telecommunications Research Center Vienna), Vienna, Austria \\ Contact: xu@ftw.at
}

\begin{abstract}
Opportunistic interference alignment (OIA) exploits channel randomness and multiuser diversity by user selection. In this paper, we address the major disadvantage of OIA which requires the feedback of the locally measured interference alignment from all users. We propose a selective feedback scheme for OIA by thresholding, where only a subset of users are required to send feedback to the transmitter. The proposed scheme can reduce the amount of feedback and still achieve the optimal degrees of freedom (DoF). We characterize the threshold and the corresponding feedback load to achieve the full DoF for a given signal-to-noise ratio. Both theoretical analysis and simulation results show that the amount of feedback can be dramatically reduced (by one order of magnitude at $20 \mathrm{~dB}$ SNR and two orders of magnitude at 30dB SNR), while still preserving the essential DoF promised by conventional OIA with full feedback.
\end{abstract}

Index Terms-Opportunistic interference alignment, user selection, random beamforming, feedback, IA.

\section{INTRODUCTION}

Interference is a crucial limitation in next generation cellular systems. To address this problem, interference alignment (IA) has attracted much attention and has been extensively studied lately. IA is able to achieve the optimal degrees of freedom (DoF) at high signal-to-noise ratio (SNR) and a rate of $M / 2 \cdot \log (\mathrm{SNR})+o(\log (\mathrm{SNR}))$ for the $M$ cell interference channel. For IA a closed-form solution of the precoding vectors for single antenna nodes with symbol extension is known [1]. However, this coding scheme is based on the assumption that global channel state information (CSI) is available at all nodes, which is extremely hard to achieve and maybe even impossible. An iterative IA algorithm is proposed in [2] to find the precoding matrices numerically with only local CSI at each node exploiting channel reciprocity. However, a number of iterations involving singular value decompositions (SVDs) have to be conducted which greatly increases the computational complexity.

For the sake of complexity reduction, opportunistic interference alignment (OIA) has been studied lately [3]-[7]. The key idea of OIA is to exploit the channel randomness and multiuser diversity by proper user selection. In [3]-[7], signal subspace dimensions are used to align the interference signals. Each transmitter opportunistically selects and serves the user whose interference channels are most aligned to each other. The degrees of alignment is quantified by a metric. To facilitate a user selection algorithm, all potential users associated with the transmitter are required to calculate and feedback the metric value based on the local CSI. Perfect IA can be achieved asymptotically with an increasing number of users. The corresponding user scaling law to obtain the optimal DoF is characterized for multiple access channels in [3], [4] and for interference channels in [6] [7]. For instance, in a 3cell $d \times 2 d$ multiple-input multiple-output (MIMO) interference channel, [7] shows that the optimal DoF $d$ is achieved if the number of users $K$ is scaled as $K \propto \mathrm{SNR}^{d^{2}}$. Therefore, at higher SNR, a larger number of users is required to achieve the optimal DoF. Clearly, the level of required total CSI feedback also increases proportionally to the number of users. However, in practical systems, the feedback is costly and the bandwidth of the feedback channel is limited. As a result, the feedback rate should be kept as small as possible.

The problem is tackled first in [8], [9] by selective feedback for single-input single-output multiuser channels. The solution is to let the users threshold their receive SNRs and notify the transmitter only if their SNR exceeds a predetermined threshold. The work can be extended to MIMO multiuser channels with random beamforming. For interference channels, this idea is mentioned in [10] for the $2 \times 2$ MIMO case using OIA. However, to the best of our knowledge, the achievability of the optimal DoF with threshold-based selective feedback is still unknown.

Can we reduce the amount of feedback and still preserve the optimal DoF? In this paper, we address this problem by selective feedback for a 3-cell MIMO interference channel. The users to be scheduled are limited to those who experience a good enough channel, thus strongly reducing the required amount of feedback.

\section{Contributions of This Paper:}

- We derive the scheduling outage probability according to the metric distribution of the best user out of total $K$ users.

- We prove that, for OIA with selective feedback, the full DoF $d$ is achieved if the scheduling outage probability scales as $\frac{1}{\mathrm{SNR}}$.

- We provide a closed form expression for setting a threshold to achieve the optimal DoF.

- We characterize the feedback load in relation to different threshold choices.

\section{SYSTEM MODEL}

Let us consider the system model for the 3-cell MIMO interference channel, as shown in Fig. 1. It consists of 3 


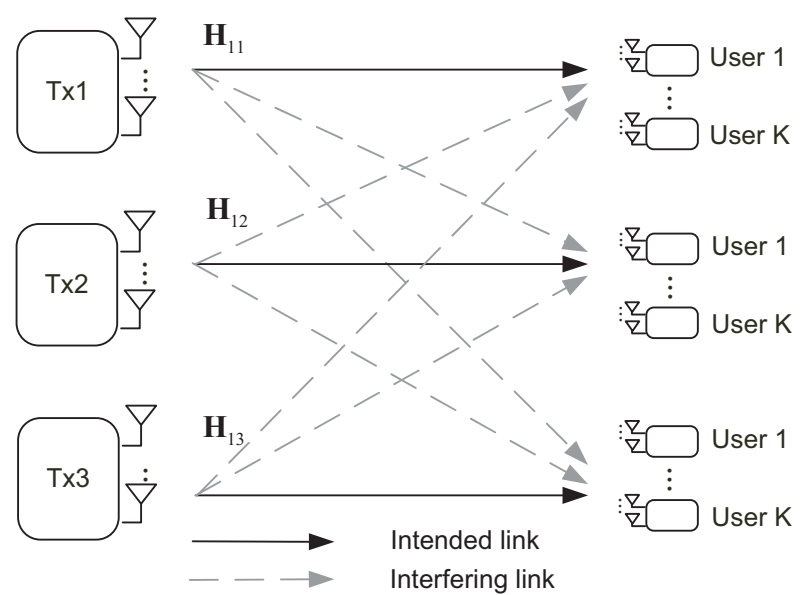

Fig. 1. Three-cell MIMO interference channel with $K$ candidates in each cell

transmitters with $N_{\mathrm{T}}$ antennas, each serving $K$ users with $N_{\mathrm{R}}$ antennas. The channel matrix from transmitter $j$ to receiver $k$ in cell $i$ is denoted by $\mathbf{H}_{i, j}^{k} \in \mathbb{C}^{N_{\mathrm{R}} \times N_{\mathrm{T}}}, \forall i, j \in\{1,2,3\}$ and $k \in\{1, \ldots, K\}$. Every element of $\mathbf{H}_{i, j}^{k}$ is an independent identically distributed (i.i.d.) symmetric complex Gaussian random variable with zero mean and unit variance.

For a given transmitter, its signal is only intended to be received and decoded by a single user for a given signaling interval. The signal received at receiver $k \in\{1, \ldots, K\}$ in cell $i$ at a given time instant is the superposition of the signals transmitted by all three transmitters, which can be written as

$$
\mathbf{x}_{i}^{k}=\mathbf{H}_{i, i}^{k} \mathbf{V}_{i} \mathbf{s}_{i}+\sum_{j=1, j \neq i}^{3} \mathbf{H}_{i, j}^{k} \mathbf{V}_{j} \mathbf{s}_{j}+\mathbf{n}_{i}^{k},
$$

where vector $\mathbf{s}_{j} \in \mathbb{C}^{d \times 1}$ denotes $d$ transmitted symbols from transmitter $j$ with power constraint $\mathbb{E}\left\{\mathbf{s}_{j} \mathbf{s}_{j}^{\mathrm{H}}\right\}=(P / d) \mathbf{I}_{d}$. In this paper, we assume $N_{\mathrm{R}}=2 d$ and $N_{\mathrm{T}} \geq d$. $\mathbf{V}_{j} \in \mathbb{C}^{N_{\mathrm{T}} \times d}$ is the corresponding linear precoding matrix. The additive complex symmetric Gaussian noise $\mathbf{n}_{i}^{k} \sim \mathcal{C N}\left(0, \mathbf{I}_{N_{\mathrm{R}}}\right)$ has zero mean and unit variance. Thus, the $\mathrm{SNR}$ becomes $\mathrm{SNR}=P$. Defining $\mathbf{U}_{i}^{k} \in \mathbb{C}^{N_{\mathrm{R}} \times d}$ as the postfiltering matrix at receiver $k$ in cell $i$, the received signal of user $k$ in cell $i$ becomes

$$
\begin{aligned}
\mathbf{y}_{i}^{k} & =\mathbf{U}_{i}^{k^{\mathrm{H}}} \mathbf{x}_{i}^{k} \\
& =\mathbf{U}_{i}^{k^{\mathrm{H}}} \overline{\mathbf{H}}_{i, i}^{k} \mathbf{s}_{i}+\sum_{j=1, j \neq i}^{3} \mathbf{U}_{i}^{k}{ }^{\mathrm{H}} \overline{\mathbf{H}}_{i, j}^{k} \mathbf{s}_{j}+\overline{\mathbf{n}}_{i}^{k} .
\end{aligned}
$$

Let $\overline{\mathbf{H}}_{i, j}^{k}=\mathbf{H}_{i, j}^{k} \mathbf{V}_{j}$ denote the effective channel and $\overline{\mathbf{n}}_{i}^{k}=$ $\mathbf{U}_{i}^{k}{ }^{\mathrm{H}} \mathbf{n}_{i}^{k}$ denote the effective spatially white noise vector. The achievable instantaneous rate for user $k$ in cell $i$ becomes

$$
\begin{aligned}
R_{i}^{k}=\log _{2} \operatorname{det} & \left(\mathbf{I}+\mathbf{U}_{i}^{k}{ }^{\mathrm{H}} \overline{\mathbf{H}}_{i, i}^{k} \overline{\mathbf{H}}_{i, i}^{k \mathrm{H}} \mathbf{U}_{i}^{k}\right. \\
& \left.\left(\sum_{j=1, j \neq i}^{3} \mathbf{U}_{i}^{k \mathrm{H}} \overline{\mathbf{H}}_{i, j}^{k} \overline{\mathbf{H}}_{i, j}^{k \mathrm{H}} \mathbf{U}_{i}^{k}+\sigma^{2} \mathbf{I}_{d}\right)^{-1}\right) .
\end{aligned}
$$
feedback algorithm.
In this section, we first derive the pre- and post-selection metric distributions, which are then used to characterize the threshold and the corresponding feedback load for our new

Without requiring global channel knowledge, OIA is able to achieve the same DoF as IA with only local CSI feedback within a cell. In this section, we describe the selection criteria and the design of the postfilter for the conventional OIA algorithm. The key idea of OIA [7] is to exploit the channel randomness and the multi-user diversity, using the following procedure:

- Each transmitter randomly picks a set of $d$ orthogonal beamforming vectors $\mathbf{v}_{j}^{1}, \ldots \mathbf{v}_{j}^{d}$, forming the truncated unitary matrix $\mathbf{V}=\left[\mathbf{v}_{j}^{1}, \ldots \mathbf{v}_{j}^{d}\right]$.

- Each user equipment measures the channel quality using a specific metric and feeds back the value of the metric to its own transmitter.

- The transmitter selects a user in its own cell for communication according to the feedback values.

Let $k^{*}$ denote the index of the selected user in cell $i$, then the average achievable sum rate becomes

$$
R_{\mathrm{sum}}=\mathbb{E}\left\{\sum_{i=1}^{3} R_{i}^{k^{*}}\right\} .
$$

The transmitters aim at choosing a user, who observes most aligned interference signals from the other transmitters. The degree of alignment is quantified by a subspace distance measure, named chordal distance. It is generally defined as

$$
d_{c}(\mathbf{A}, \mathbf{B})=1 / \sqrt{2}\left\|\mathbf{A} \mathbf{A}^{\mathrm{H}}-\mathbf{B} \mathbf{B}^{\mathrm{H}}\right\|_{\mathrm{F}}
$$

where $\mathbf{A}, \mathbf{B} \in \mathbb{C}^{N_{\mathrm{R}} \times d}$ are the orthonormal bases of two subspaces. For OIA, each user finds an orthonormal basis $\mathbf{Q}$ of the column space spanned by the the two interference channels respectively, i.e., $\mathbf{Q}_{i p}^{k} \in \operatorname{span}\left(\hat{\mathbf{H}}_{i p}^{k}\right)$ and $\mathbf{Q}_{i q}^{k} \in \operatorname{span}\left(\hat{\mathbf{H}}_{i q}^{k}\right)$ where $p=(i+1 \bmod 3)$ and $q=(i+2 \bmod 3)$. Then the users calculate the distance between two interference subspaces using the obtained orthonormal basis, yielding

$$
\mathcal{D}_{i}^{k}=d_{\mathrm{c}}^{2}\left(\mathbf{Q}_{i p}^{k}, \mathbf{Q}_{i q}^{k}\right),
$$

where $\mathcal{D}_{i}^{k}$ is the distance measured at user $k$ in cell $i$. For conventional OIA, all users feed back the distance measure to the transmitters and the user selected by transmitter $i$ is given by

$$
k^{*}=\arg \min _{k} \mathcal{D}_{i}^{k}
$$

The metric value of the selected user is given by $\mathcal{D}_{i}^{k^{*}}$.

\section{Threshold-BAsed Selective Feedback for OIA}




\section{A. Metric Distribution}

As shown in [11], for quantizing a source arbitrarily distributed on the Grassmannian manifold $\mathcal{G}_{N_{\mathrm{R}}, d}(\mathbb{C})$ using a random codebook with $K$ codewords, the second moment of the chordal distance can be bounded as

$$
\begin{aligned}
Q(K) & =\mathbb{E}\left[\min _{\mathbf{C}_{k} \in \mathcal{C}_{\mathrm{rnd}}} d_{\mathrm{c}}^{2}\left(\mathbf{A}, \mathbf{C}_{k}\right)\right] \\
& \leq \frac{\Gamma\left(\frac{1}{d\left(N_{\mathrm{R}}-d\right)}\right)}{d\left(N_{\mathrm{R}}-d\right)}\left(K c_{N_{\mathrm{R}}, d}\right)^{-\frac{1}{d\left(N_{\mathrm{R}}-d\right)}}
\end{aligned}
$$

where $\Gamma(\cdot)$ denotes the Gamma function and the random codebook $\mathcal{C}_{\text {rnd }} \subset \mathcal{G}_{N_{\mathrm{R}}, d}(\mathbb{C})$. The constant $c_{N_{\mathrm{R}}, d}$ is the ball volume on the Grassmannian manifold $\mathcal{G}_{N_{\mathrm{R}}, d}(\mathbb{C})$, i.e.

$$
c_{N_{\mathrm{R}}, d}=\frac{1}{\Gamma\left(d\left(N_{\mathrm{R}}-d\right)+1\right)} \prod_{i=1}^{d} \frac{\Gamma\left(N_{\mathrm{R}}-i+1\right)}{\Gamma(d-i+1)} .
$$

The problem of selecting the best user out of $K$ users is equivalent to quantizing an arbitrary subspace with $K$ random subspaces on the Grassmannian manifold $\mathcal{G}_{N_{\mathrm{R}}, d}(\mathbb{C})$ [7, Lemma 4]. Therefore, we have $\mathbb{E}\left[\mathcal{D}_{i}^{k}\right]=Q(1)$ and $\mathbb{E}\left[\mathcal{D}_{i}^{k^{*}}\right]=Q(K)$.

The cumulative density function $(\mathrm{CDF})$ of $\mathcal{D}_{i}^{k}$, defined as $F_{\mathcal{D}}(x)$, is given by [11]

$$
\begin{aligned}
F_{\mathcal{D}}(x) & =\operatorname{Pr}\left(\mathcal{D}_{i}^{k} \leq x\right) \\
& =\operatorname{Pr}\left(d_{\mathrm{c}}^{2}\left(\mathbf{A}, \mathbf{C}_{k}\right) \leq x\right) \\
& = \begin{cases}0, & x<0 \\
c_{N_{\mathrm{R}}, d} \cdot x^{d\left(N_{\mathrm{R}}-d\right)}, & 0 \leq x \leq \hat{x} \\
1, & x>\hat{x}\end{cases}
\end{aligned}
$$

where $\hat{x}$ is the upper bound of $x$ satisfying $c_{N_{\mathrm{R}}, d} \cdot \hat{x}^{d\left(N_{\mathrm{R}}-d\right)}=$ 1. For the selected user, the CDF of $\mathcal{D}_{i}^{k^{*}}$, defined as $F_{\mathcal{D}}^{K}(x)$, corresponds to the complement of the event where all $K$ users exceed $x$, i.e.,

$$
\begin{aligned}
F_{\mathcal{D}}^{K}(x) & =\operatorname{Pr}\left(\mathcal{D}_{i}^{k^{*}} \leq x\right) \\
& =\operatorname{Pr}\left(\min _{\mathbf{C}_{k} \in \mathcal{C}_{\text {rnd }}} d_{\mathrm{c}}^{2}\left(\mathbf{A}, \mathbf{C}_{k}\right) \leq x\right) \\
& =1-\left(1-F_{\mathcal{D}}(x)\right)^{K} .
\end{aligned}
$$

\section{B. Achievable DoF of Conventional OIA}

We briefly revisit the results obtained in [7], which will be used for comparison with our new scheme. A finite number of users $K$ results in residual interference. Let us define the rate loss term due to residual interference as

$$
R_{\mathrm{loss} i}^{k}=\log _{2} \operatorname{det}\left(\sum_{j=1, j \neq i}^{3} \mathbf{U}_{i}^{k \mathrm{H}} \overline{\mathbf{H}}_{i, j}^{k} \overline{\mathbf{H}}_{i, j}^{k \mathrm{H}} \mathbf{U}_{i}^{k}+\sigma^{2} \mathbf{I}_{d}\right) \text {. }
$$

When the cell $i$ has $K$ users, the average rate loss at the selected user $k^{*}$ can be bounded as

$$
\begin{aligned}
\mathbb{E}\left[R_{\operatorname{loss} i}^{k^{*}}\right] & \leq d \cdot \log _{2}\left(1+\frac{P}{d} \cdot \mathbb{E}\left[\mathcal{D}_{i}^{k^{*}}\right]\right) \\
& =d \cdot \log _{2}\left(1+\frac{P}{d} \cdot Q(K)\right),
\end{aligned}
$$

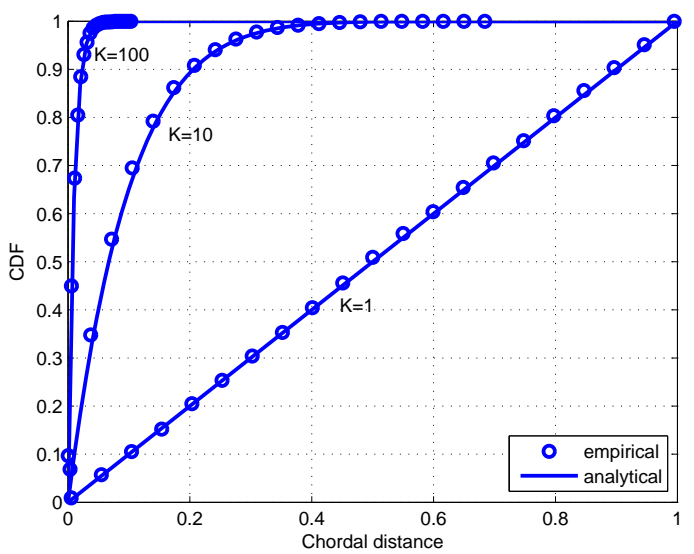

Fig. 2. The CDF of $\mathcal{D}_{i}^{k^{*}}$ with varying number of users $K$ in each cell. The number of receive antennas $N_{\mathrm{R}}=2$. The number of transmitted symbols $d=1$.

where (18) and (19) are obtained due to [7, Lemma 6].

The achievable DoF of transmitter $i$ using OIA can be expressed by $d-\lim _{P \rightarrow \infty} \frac{\mathbb{E}\left[R_{\operatorname{logs}_{i}} k^{*}\right]}{\log _{2} P}$. The full DoF $d$ is achieved if the number of users is scaled as [7, Theorem 2]

$$
K \propto P^{d^{2}} .
$$

\section{Selective Feedback by Thresholding}

For OIA, the user selected for transmission is the one with the smallest chordal distance measure. For a reasonable number of users $K$, it can rarely happen that a user with a "bad" channel will be selected by the transmitter. Therefore, the feedback channel bandwidth provisioned for such a user is wasted. In fact, only the users experiencing good enough conditions have a good chance to be selected and should feedback their channel quality. To this end, we propose a threshold-based feedback strategy where only a subset of users, whose chordal distance measure is smaller than a predetermined threshold, send feedback to the transmitter. Importantly, users decide locally whether they should attempt to access the channel and send feedback to the transmitter or not. Hence, only a fraction of the users are required to send feedback and the required bandwidth of the feedback channel can be reduced substantially.

Fig. 2 shows the $\mathrm{CDF}$ of $\mathcal{D}_{i}^{k^{*}}$ with $N_{\mathrm{R}}=2$ receive antennas and $d=1$ transmitted symbols. The analytical and empirical results are presented with different number of users $K \in\{1,10,100\}$. It can be observed that the analytical results obtained from (16) agree perfectly with empirical results. When $K=1, F_{\mathcal{D}}^{K}(x)=F_{\mathcal{D}}(x)$ is the $\mathrm{CDF}$ of chordal distance before user selection. The chordal distance measure of the best user $\mathcal{D}_{i}^{k^{*}}$ becomes smaller with the increasing $K$. Intuitively speaking, with a relatively large $K$, the chordal distance measure of the best user is very small and has a more concentrated distribution, as shown in Fig. 2. This observation gives rise to our proposed selective feedback scheme for OIA. For instance, When $K=10$, a threshold of 0.4 guarantees 
with nearly probability one that the best user would fall below the threshold and sends feedback to the transmitter. Therefore, only $40 \%$ of the users will feed back a value to the transmitter (since $F_{\mathcal{D}}(x)=x$ for $0 \leq x \leq \hat{x}$ when $N_{\mathrm{R}}=2$ and $d=1$ ). On the other hand, a scheduling outage occurs if no user sends feedback to the transmitter. In such an event, a random user will be selected.

OIA is able to achieve the optimal DoF, if the number of users in each cell scales with $P^{d^{2}}$. For threshold-based OIA, it is unknown how to set a threshold such that the DoF is still achievable. In this section, we characterize the threshold as a function of the transmit power for different MIMO configurations. We define the probability density functions (PDFs) of $\mathcal{D}_{i}^{k}$ and $\mathcal{D}_{i}^{k^{*}}$ as $f_{\mathcal{D}}(x)$ and $f_{\mathcal{D}}^{K}(x)$ respectively, where $\int_{-\infty}^{x} f_{\mathcal{D}}(x) \mathrm{d} x=F_{\mathcal{D}}(x)$ and $\int_{-\infty}^{x} f_{\mathcal{D}}^{K}(x) \mathrm{d} x=F_{\mathcal{D}}^{K}(x)$. In order to distinguish from the previous conventional OIA, we employ $k^{\dagger}$ as the index of the selected user with thresholdbased selective feedback. Denoting the feedback threshold by $x_{\mathrm{th}}$, the expected metric value of the selected user $k^{\dagger}$ can be expressed as

$$
\begin{aligned}
\mathbb{E}\left[\mathcal{D}_{i}^{\left.k^{\dagger}\right]}=\right. & F_{\mathcal{D}}^{K}\left(x_{\mathrm{th}}\right) \frac{\int_{0}^{x_{\mathrm{th}}} f_{\mathcal{D}}^{K}(x) x \mathrm{~d} x}{F_{\mathcal{D}}^{K}\left(x_{\mathrm{th}}\right)} \\
& +\left(1-F_{\mathcal{D}}^{K}\left(x_{\mathrm{th}}\right)\right) \frac{\int_{x_{\mathrm{th}}}^{\hat{x}} f_{\mathcal{D}}(x) x \mathrm{~d} x}{1-F_{\mathcal{D}}\left(x_{\mathrm{th}}\right)} \\
\leq & \int_{0}^{\hat{x}} f_{\mathcal{D}}^{K}(x) x \mathrm{~d} x+\left(1-F_{\mathcal{D}}^{K}\left(x_{\mathrm{th}}\right)\right) \frac{\int_{x_{\mathrm{th}}}^{\hat{x}} f_{\mathcal{D}}(x) x \mathrm{~d} x}{1-F_{\mathcal{D}}\left(x_{\mathrm{th}}\right)} \\
\leq & \int_{0}^{\hat{x}} f_{\mathcal{D}}^{K}(x) x \mathrm{~d} x+\left(1-F_{\mathcal{D}}^{K}\left(x_{\mathrm{th}}\right)\right) \hat{x} \\
= & Q(K)+\left(1-F_{\mathcal{D}}^{K}\left(x_{\mathrm{th}}\right)\right) \hat{x}
\end{aligned}
$$

where $\frac{f_{\mathcal{D}}(x)}{1-F_{\mathcal{D}}\left(x_{\mathrm{th}}\right)}$ and $\frac{f_{\mathcal{D}}^{K}(x)}{F_{\mathcal{D}}^{K}\left(x_{\mathrm{th}}\right)}$ are the normalized truncated PDFs of $\mathcal{D}_{i}^{k}$ and $\mathcal{D}_{i}^{k^{*}}$ satisfying $\frac{\int_{x_{\mathrm{th}}}^{\hat{x}} f_{\mathcal{D}}(x) \mathrm{d} x}{1-F_{\mathcal{D}}\left(x_{\mathrm{th}}\right)}=1$ and $\frac{\int_{0}^{x_{\mathrm{th}}} f_{\mathcal{D}}^{K}(x) \mathrm{d} x}{F_{\mathcal{D}}^{K}\left(x_{\mathrm{th}}\right)}=1$. The first term in (21) represents the event where the selected user falls below the threshold and the second term denotes a scheduling outage. Equation (22) follows from the fact $0 \leq x_{\text {th }} \leq \hat{x}$. Equation (23) is obtained by taking the upper limit of the integration. Since the achievable DoF of transmitter $i$ is $d-\lim _{P \rightarrow \infty} \frac{\mathbb{E}\left[R_{\operatorname{loss}^{*}}{ }^{*}\right]}{\log _{2} P}$, applying the upper bound derived in (24) to (18), the full DoF $d$ is achieved if $K \propto P^{d^{2}}$ and the scheduling outage probability

$$
\left(1-F_{\mathcal{D}}^{K}\left(x_{\mathrm{th}}\right)\right) \propto \frac{1}{P} \Leftrightarrow\left(1-F_{\mathcal{D}}^{K}\left(x_{\mathrm{th}}\right)\right)=\frac{\alpha}{P}
$$

where $\alpha$ is a positive constant. Therefore, the average rate loss $\mathbb{E}\left[R_{\operatorname{loss} i}^{k^{*}}\right]$ is upper bounded by a constant when $P \rightarrow \infty$. By taking the equality of (25) with $\alpha=1$ and using the results obtained in (16) and (13), the threshold which achieves the full DoF $d$ is given by

$$
x_{\mathrm{th}}=\left(\frac{1-P^{\frac{-1}{K}}}{c_{N_{\mathrm{R}}, d}}\right)^{\frac{1}{d\left(N_{\mathrm{R}}-d\right)}} .
$$

\section{Average Feedback Load}

We are interested in characterizing the required feedback load to achieve the full DoF using the proposed thresholdbased selective feedback for OIA. Let us define the average feedback load $N$ as the average number of feedback users per cell. The normalized feedback load $n$ is the ratio of the average feedback load $N$ to the total number of users $K$, which is equal to $n=F_{\mathcal{D}}\left(x_{\mathrm{th}}\right)$. Invoking the result obtained from (16) to (25), the normalized feedback load achieving full DoF $d$ is given by

$$
n=F_{\mathcal{D}}\left(x_{\mathrm{th}}\right)= \begin{cases}0, & x_{\mathrm{th}}<0 \\ 1-P^{\frac{-1}{K}}, & 0 \leq x_{\mathrm{th}} \leq \hat{x} \\ 1, & x_{\mathrm{th}}>\hat{x}\end{cases}
$$

Therefore, the average feedback load $N$ can be calculated as

$$
N=n K=K\left(1-P^{\frac{-1}{K}}\right) .
$$

\section{E. Other Threshold Choices for OIA}

The choice of the threshold $x_{\text {th }}$ can be made according to various criteria. The threshold which satisfies a given feedback ratio $n$ is given by

$$
x_{\mathrm{th}}=\left(\frac{n}{c_{N_{\mathrm{R}}, d}}\right)^{\frac{1}{d\left(N_{\mathrm{R}}-d\right)}} .
$$

In case if the capacity of the feedback channel is limited, the threshold satisfying a given total feedback load $N$ is given by

$$
\begin{aligned}
& x_{\mathrm{th}}=\left(\frac{N}{K c_{N_{\mathrm{R}}, d}}\right)^{\frac{1}{d\left(N_{\mathrm{R}}-d\right)}} . \\
& \text { V. SIMULATION RESULTS }
\end{aligned}
$$

In this section, we provide numerical results of OIA in terms of sum rate using the feedback approaches abbreviated as follows:

- OIA-F: OIA with full feedback [7]

- OIA-S: OIA with our new threshold-based selective feedback

Fig. 3 shows the achievable sum rate versus SNR of the above schemes with $N_{\mathrm{R}}=N_{\mathrm{T}}=2, d=1$ and the number of users $K=P^{d^{2}}$. The thresholds $x_{\mathrm{th}}=\{0,0.305,0.206,0.103,0.045,0.018,0.007\}$ are adaptively chosen at different SNRs according to (26). We can see that OIA with selective feedback achieves almost the same performance as OIA with full feedback. Both of these two schemes achieve DoF $d=1$ compared to the reference line. However, by using the adaptive threshold (26), we can reduce the feedback significantly, which can be seen in Fig. 4. One interesting observation is that the feedback load grows very slightly with the increase of SNR. At SNR $=30 \mathrm{~dB}$, the average feedback load is reduced to less than $1 \%$ of the full feedback OIA. In addition, the analytical results from (28) perfectly matches to the simulation results.

Fig. 5 shows the achievable sum rate as a function of the normalized feedback load $n$ with $N_{\mathrm{R}}=N_{\mathrm{T}}=2, d=1$ and 


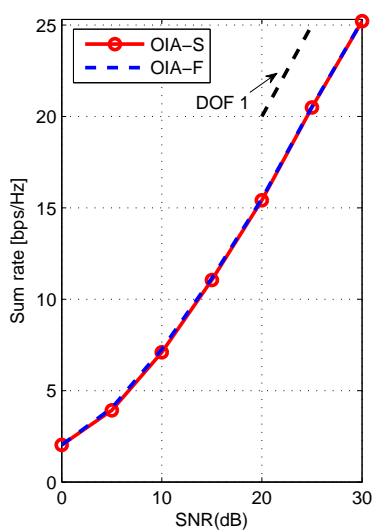

Fig. 3. Achievable sum rate for $N_{\mathrm{R}}=N_{\mathrm{T}}=2, d=1$ and $K=P^{d^{2}}$.

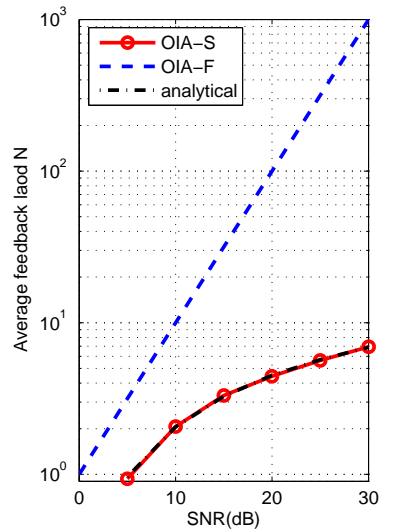

Fig. 4. Average feedback load for $N_{\mathrm{R}_{2}}=N_{\mathrm{T}}=2, d=1$ and $K=$ $P^{d^{2}}$.

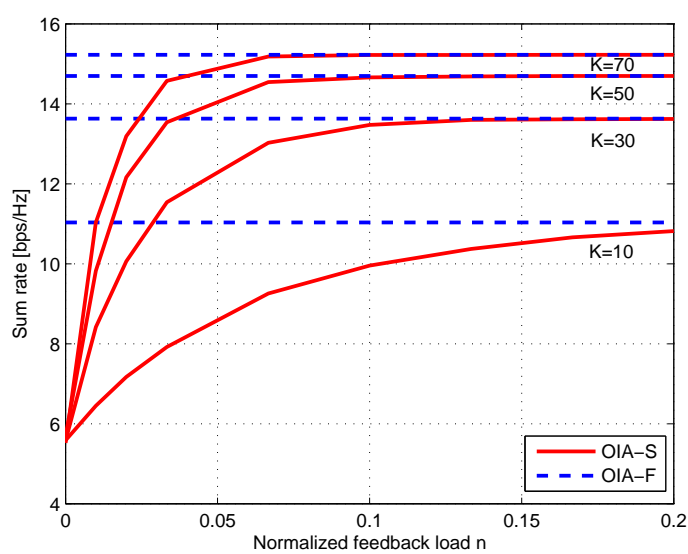

Fig. 5. Achievable sum rate at $\mathrm{SNR}=20 \mathrm{~dB}$ for $N_{\mathrm{R}}=N_{\mathrm{T}}=2, d=1$ and $K \in\{10,30,50,70\}$.

the number of users $K \in\{10,30,50,70\}$. The corresponding thresholds are calculated using (29). We see that for $K \geq 30$, a $10 \%$ feedback load results in a negligible loss in sum rate.

In Fig. 6, the achievable sum rate is evaluated with $N_{\mathrm{R}}=$ $N_{\mathrm{T}}=4, d=2$ and $K \in\{10,50,100\}$. For OIA with selective feedback, a feedback load of $n=10 \%$ is used. The corresponding thresholds are obtained using (29). With a finite number of users, interference is inevitably leaked to the signal subspace. We can observed that the sum rate increases as the number of users increases. For $K \in\{50,100\}$, OIA with $10 \%$ feedback load guarantees almost the same performance as OIA with full feedback.

\section{CONCLUSION}

In this paper, we proposed a threshold-based selective feedback scheme for OIA, where only a subset of users are required to send feedback to the transmitter. We characterized the threshold and the corresponding feedback load achieving the full DoF at different signal-to-noise ratios. Both theoretical analysis and simulation results showed that the amount of

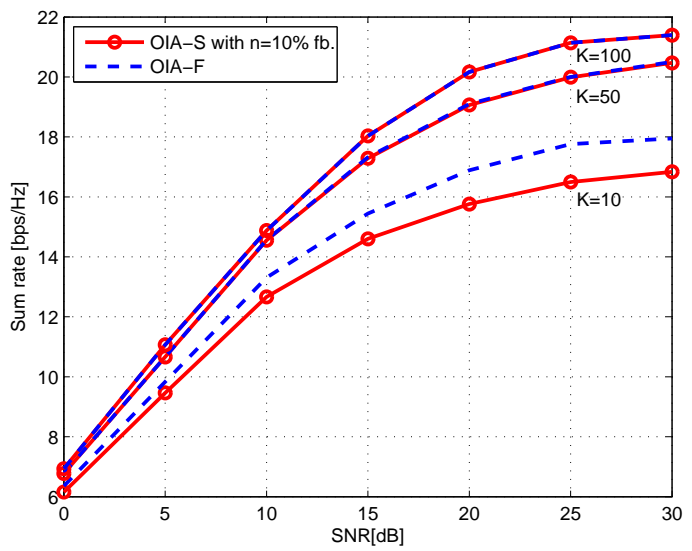

Fig. 6. Achievable sum rate for $N_{\mathrm{R}}=N_{\mathrm{T}}=4, d=2$ and $K \in$ $\{10,50,100\}$.

feedback can be dramatically reduced by more than one magnitude, while preserving the essential DoF promised by conventional OIA with full feedback.

\section{ACKNOWLEDGMENT}

This work was supported by the FTW project I-0. The Telecommunications Research Center Vienna (FTW) is supported by the Austrian Government and the City of Vienna within the competence center program COMET.

\section{REFERENCES}

[1] V. Cadambe and S. Jafar, "Interference Alignment and Degrees of Freedom of the K-User Interference Channel," IEEE Trans. Inf. Theory, vol. 54, no. 8, pp. 3425-3441, Aug. 2008.

[2] K. Gomadam, V. R. Cadambe, and S. A. Jafar, "A distributed numerical approach to interference alignment and applications to wireless interference networks," IEEE Trans. Inf. Theory, vol. 57, no. 6, pp. 3309-3322, Jun. 2011.

[3] H. J. Yang, W.-y. Shin, B. C. Jung, and A. Paulraj, "Opportunistic interference alignment for MIMO interfering multiple-access channels,' IEEE Trans. Wirel. Commun., vol. 12, no. 5, pp. 2180-2192, May 2013.

[4] B. C. Jung, D. Park, and W. Shin, "Opportunistic interference mitigation achieves optimal degrees-of-freedom in wireless multi-cell uplink networks," IEEE Trans. Commun., vol. 60, no. 7, pp. 1935-1944, Jul. 2012.

[5] T. Gou, T. Koike-Akino, and P. Orlik, "Improved and opportunistic interference alignment schemes for multi-cell interference channels," in Proc. IEEE Veh. Technol. Conf. IEEE, May 2012, pp. 1-5.

[6] J. H. Lee, W. Choi, and B. D. Rao, "Multiuser diversity in interfering broadcast channels: achievable degrees of freedom and user scaling law," IEEE Trans. Wirel. Commun., vol. 12, no. 11, pp. 5837-5849, Nov. 2013.

[7] J. H. Lee and W. Choi, "On the achievable DoF and user scaling law of opportunistic interference alignment in 3-transmitter MIMO interference channels," IEEE Trans. Wirel. Commun., vol. 12, no. 6, pp. 2743-2753, Jun. 2013.

[8] D. Gesbert and M.-S. Alouini, "Selective multi-user diversity," in Proc. 3rd IEEE Int. Symp. Signal Process. Inf. Technol., 2003, pp. 162-165.

[9] D. Gesbert and M. Alouini, "How much feedback is multi-user diversity really worth?" in Proc. IEEE Int. Conf. Commun. IEEE, 2004, pp. 234238.

[10] H. Liu, H. Gao, W. Long, and T. Lv, "A novel ccheme for downlink opportunistic interference alignment," Mar. 2014. [Online]. Available: http://arxiv.org/abs/1403.3978

[11] W. Dai, Y. E. Liu, and B. Rider, "Quantization bounds on Grassmann manifolds and applications to MIMO communications," IEEE Trans. Inf. Theory, vol. 54, no. 3, pp. 1108-1123, Mar. 2008. 